\title{
Impact of Interprofessional Collaborative Practice in Palliative Care on Outcomes for Advanced Cancer Inpatients in Resource Limited Setting
}

Pitchayapa Pornrattanakavee

Phramongkutklao Hospital

\section{Tassaya Srichan}

Phramongkutklao Hospital

\section{Kasan Seetalarom}

Phramongkutklao Hospital

\section{Siriwimon Saichamchan}

Phramongkutklao Hospital

\section{Nittha Oer-areemitr}

Phramongkutklao Hospital

Naiyarat Prasongsook ( $\nabla$ naiyarat_p@yahoo.co.th )

Phramongkutklao Hospital

\section{Research Article}

Keywords: advanced cancer inpatients, interprofessional collaborative practice, team-based approach, palliative care, specialty palliative care, palliative care team, quality of life (QoL), re-admission, anxiety, depression

Posted Date: February 21st, 2022

DOI: https://doi.org/10.21203/rs.3.rs-1132174/v1

License: (a) (1) This work is licensed under a Creative Commons Attribution 4.0 International License. Read Full License 


\section{Abstract \\ Background}

Palliative care for advanced cancer patients improves suffering symptoms, quality of life (QoLs).

However, routine implementation of palliative care by specialty palliative care consultation is still unmet need among advanced cancer inpatients. Our study aim is to evaluate the effectiveness of team-based approach on QoLs and readmission rate when compare to routine practice by among medical oncologists.

\section{Methods}

This study was prospective, Quasi-Experimental design. Advanced cancer inpatients were non-randomly assigned to receive palliative care service by team-based approach or medical oncologists only. The primary endpoint was QoL. The secondary endpoint was readmission rate at 7, and 30 days of hospital discharge.

\section{Results}

One hundred twenty-two inpatients were enrolled. Inpatients who were assessed by team-based approach had significantly improved in change scores of subjective well-being (SWB) when compare to another group ( $\triangle$ SWB: -1 [-19-11] vs 0 [-9-15], $p$-value $=0.043$ ). Furthermore, inpatients who were assessed by team-based approach had significantly decreased in term of readmission rate at 7 days of hospital discharge (4.92\% in team-based approach group vs. $19.67 \%$ in medical oncologist group, $p$-value= 0.013).

\section{Conclusions}

Interdisciplinary collaboration is key success for establishing goal of care, which are supporting the best possible QoL and relieving suffering symptoms for those advanced cancer inpatients. Furthermore, readmission rate at 7 days of hospital discharge was significantly reduced by team-based approach. Therefore, comprehensive palliative care assessment by interprofessional collaborative practice is required.

\section{Background}

Integration of palliative care in cancer patients improve oncology outcomes, including suffering symptoms, quality of life, and survival. More than 40 million people each year are expected to be needed for palliative care (1). Furthermore, most of them (78\%) live in low- and middle-income countries. 
However, only $14 \%$ of those people will be received comprehensive palliative care (1) due to limitedresources available for palliative care specialists particularly.

Systematic review demonstrated that the best outcomes with palliative care was provided by an interprofessional palliative care team and should be initiated within 8 weeks of diagnosis (2). For example, a meta-analysis results showed a $14 \%$ increase in 1-year survival, and median overall survival benefit of 4.56 months in patients with advanced cancer who were received palliative care by outpatient specialty palliative care team. Moreover, quality of life and cost-effectiveness were improved significantly. Regarding to recent data, comprehensive palliative care in advanced cancer patients by interprofessional palliative care team can be favorably impact health systems and cost-effective (3-4).

Multidisciplinary team including board-certified palliative care physicians, advanced practice nurses, physician assistants, nurses, dieticians, social workers, and pharmacists is required for providing direct care to patients, families, caregivers (2). In fact, the most of advanced cancer inpatients who are needed with palliative care approach in clinical practice are managed by medical oncologist alone, which palliative and oncologic aspects of care might have an inferiority for placing the entire burden on an interprofessional team approach. Therefore, a novel model of palliative care service delivery requires to be developed in limited-resources. In this study, we designed a novel model for the inpatient hospital setting, which was a palliative care nurse and medical oncology co-working model of care.

The objective of this study was to obtain the effect of the palliative care nurse and medical oncology coworking model on quality of life of advanced cancer inpatients, and readmission rate at 7 days and 30 days of hospital discharge.

\section{Patients And Methods}

\section{Study Design and Patient Selection}

This study is a prospective, single institute, non-randomized interventional study design (QuasiExperimental design) investigating the effect of interprofessional cooperative practice in palliative care on oncologic, and palliative outcomes in advanced cancer inpatients.

The trial was conducted according to ICH-GCP guidelines and the Declaration of Helsinki. The study protocol was approved by Institutional Review Boards, Royal Thai Army Medical Department. All patients provided written informed consent for trial participation. It is registered under Thai Clinical Trials Registry (TCTR20200312001). Date of first registration was March 12, 2020.

All patients with advanced stage of solid cancer $\geq 18$ years who were hospitalized due to symptomatic management, any ECOG performance status were eligible. In addition, Patients were not received any specific cancer treatment, and be able to understand and fill the questionnaires. 


\section{Treatment}

Eligible patients were non-randomly assigned to received palliative care by either interprofessional collaborative team, including specialist palliative care nurses and medical oncologists or medical oncologist alone by primary physicians. All advanced cancer inpatients were assessed and received treatment for suffering symptoms by medical oncologists. Advanced cancer in-patients who were assigned to receive palliative care by interprofessional collaborative team were evaluated and received treatment for physical-, psychological-, and spiritual aspects by both specialist palliative care nurse and medical oncologist. The palliative care visiting programs were adapted from the National Consensus Project for Quality Palliative Care (6) by using medical records and questionnaire for assessment at 1 day and 7 days after hospitalization. Both physical and psychological aspects were accessed via through medical records, and questionnaires, respectively. (Fig. 1, Supplementary Fig. 1)

\section{Patient-reported Measurements}

For psychological aspects, we used Thai-Hospital Anxiety and Depression Scale (Thai-HADS) for assessment. The Thai-HADS is composed of 14-items, which it is divided into 2 subscales such as symptoms of anxiety scores and depression scores, each of which scores a symptom severity between a 0 and 21. For each item, a score of 0 typically indicates no distress, or no anxiety, while a higher score is indicative of higher distress/ or anxiety. If score higher than 7 indicates clinically significant of distress/ or anxiety.

Health-related quality of life was measured by using the Thai- Functional Assessment of Cancer TherapyGeneral (Thai-FACT-G) scale. The main purpose of Thai FACT-G is to evaluate multiple dimensions of quality of life such as physical well-being, social/family well-being, emotional well-being and functional well-being, which scores a well-being between a 0 and 108. The higher score is indicative of improvement of quality of life.

\section{Objectives And End Points}

The primary objective of the study was to compare the quality of life (QoL) between advanced cancer inpatients who were received palliative care by interprofessional collaborative team and among medical oncologists. The secondary objective was to compare the re-admission rate at 7 days and 30 days after hospital discharge between two groups.

\section{Sample Size Calculation}

Regarding to the previous pilot study results (11), our hypothesis was that team-based approach was associated with better outcomes in QoL, and 10\% shorter hospital length of stay, and readmission rate. 
Therefore, the calculated sample was 60 patients in each arm.

\section{Statistical Analysis}

Descriptive statistics were used to estimate the frequencies, means, and standard deviation of the study variables. Differences between two groups were analyzed by using Chi-square test or Fisher's exact test. Paired T- test was used for analysis of difference in mean for Thai -FACT - General, Thai-HADS score on each group. Mann-Whitney $U$ test was used for analysis of difference in mean for Thai-FACT-General, Thai- HADS score between two groups. For all other tests, the alpha was set to 0.05 (two-sided). All statistical analyses were performed using SPSS, software, version 22. Data cutoff date was January 14, 2021 due to reaching the planned sample size. For all outcomes, the intention-to-treat analysis was conducted.

\section{Results}

\section{Patients and Treatment}

Between March and December 2020, we enrolled 122 patients who were diagnosed with advanced solid cancer without receiving any specific cancer treatment, and were hospitalized for symptomatic treatment in Phramongkutklao Hospital. Sixty-one patients were received palliative care treatment by specialist palliative care nurse and medical oncologist. Another 61 patients were evaluated and treated by medical oncologists. The baseline characteristics were similar between two groups, except metastatic site at liver and cancer pain aspect (Table 1$)$. The majority were enrolled with male (68\%), primary lung cancer $(28.7 \%)$, and cancer pain (58.1\%). The median age of patients who were received palliative care treatment by interprofessional collaborative team, and medical oncologist alone were 63, and 59 years-old, respectively. (Table 1 ) 
Table 1

Baseline characteristics of the study population

$\begin{array}{llll}\begin{array}{l}\text { Professional Collaborative } \\ \text { Team }(\mathrm{n}=61)\end{array} & \begin{array}{l}\text { Medical } \\ \text { Oncologists }(\mathrm{n}= \\ 61)\end{array} & \text { Total } & \begin{array}{l}p \text { - } \\ \text { value }\end{array} \\ \mathrm{n}(\%) & \mathrm{n}(\%) & \mathrm{n}(\%) & \end{array}$

\section{Gender}

Male

$42(68.85)$

$41(67.21)$

83

$(68.03)$

Female

$19(31.15)$

$20(32.79)$

39

(31.97)

\section{Age}

Median (Min - Max)

$63(20-88)$

$59(22-81)$

$0.862 \ddagger$

date admit

Median (Min - Max)

$14(7-127)$

$11(7-45)$

$0.012 \ddagger$

Type of Cancer

\begin{tabular}{|c|c|c|c|c|}
\hline Primary Lung Cancer & $14(22.95)$ & $21(34.43)$ & $\begin{array}{l}35 \\
(28.68)\end{array}$ & 0.161 \\
\hline Hepatocellular Carcinoma & $11(18.03)$ & $3(4.92)$ & $\begin{array}{l}14 \\
(11.47)\end{array}$ & 0.023 \\
\hline $\begin{array}{l}\text { Esophagus/Gastric/billiary } \\
\text { tract cancer }\end{array}$ & $11(18.03)$ & $12(19.67)$ & $\begin{array}{l}23 \\
(18.85)\end{array}$ & 0.817 \\
\hline Head and Neck Cancer & $4(6.56)$ & $9(14.75)$ & $\begin{array}{l}13 \\
(10.65)\end{array}$ & 0.142 \\
\hline Colon Cancer & $4(6.56)$ & $6(9.84)$ & $\begin{array}{l}10 \\
(8.19)\end{array}$ & 0.509 \\
\hline Genitourinary Cancer & $6(9.84)$ & $2(3.28)$ & $\begin{array}{l}8 \\
(4.91)\end{array}$ & $0.272 \dagger$ \\
\hline Breast Cancer & $4(6.56)$ & $4(6.56)$ & $\begin{array}{l}8 \\
(6.55)\end{array}$ & 1.000 \\
\hline Musculoskeletal Cancer & $4(6.56)$ & $1(1.64)$ & $\begin{array}{l}5 \\
(4.09)\end{array}$ & $0.365 t$ \\
\hline Two primary cancer & $2(3.28)$ & $2(3.28)$ & $\begin{array}{l}4 \\
(3.27)\end{array}$ & 1.000 \\
\hline Gynecologic Cancer & $1(1.64)$ & $1(1.64)$ & $\begin{array}{l}2 \\
(1.63)\end{array}$ & $1.000 t$ \\
\hline
\end{tabular}




\begin{tabular}{|c|c|c|c|c|}
\hline & $\begin{array}{l}\text { Professional Collaborative } \\
\text { Team }(n=61)\end{array}$ & $\begin{array}{l}\text { Medical } \\
\text { Oncologists ( } n= \\
61 \text { ) }\end{array}$ & Total & $\begin{array}{l}p- \\
\text { value }\end{array}$ \\
\hline $\begin{array}{l}\text { Brain Metastasis at } \\
\text { Admission }\end{array}$ & $6(9.84)$ & $9(14.75)$ & $\begin{array}{l}15 \\
(12.29)\end{array}$ & 0.408 \\
\hline $\begin{array}{l}\text { Liver Metastasis at } \\
\text { Admission }\end{array}$ & $27(44.26)$ & $13(21.31)$ & $\begin{array}{l}40 \\
(32.78)\end{array}$ & 0.007 \\
\hline \multicolumn{5}{|l|}{ Type of Treatment } \\
\hline Chemotherapy & $38(62.30)$ & $38(62.30)$ & $\begin{array}{l}76 \\
(62.3)\end{array}$ & 1.000 \\
\hline Surgery & 19 (31.15) & $18(29.51)$ & $\begin{array}{l}37 \\
(30.3)\end{array}$ & 0.844 \\
\hline Immunotherapy/Targeted & $7(11.48)$ & 10 (16.39) & $\begin{array}{l}17 \\
(13.4)\end{array}$ & 0.433 \\
\hline Hormonal therapy & $5(8.20)$ & $1(1.64)$ & $6(4.9)$ & $0.207 \dagger$ \\
\hline \multicolumn{5}{|l|}{ Type of Problems } \\
\hline Pain & 45 (73.77) & $26(43.33)$ & $\begin{array}{l}71 \\
(58.19)\end{array}$ & 0.001 \\
\hline Dyspnea & $21(34.43)$ & $29(47.54)$ & $\begin{array}{l}50 \\
(40.98)\end{array}$ & 0.141 \\
\hline Fatigue & $23(37.70)$ & $16(26.23)$ & $\begin{array}{l}39 \\
(31.96)\end{array}$ & 0.174 \\
\hline Abdominal Pain & $25(40.98)$ & $17(27.87)$ & $\begin{array}{l}42 \\
(34.42)\end{array}$ & 0.127 \\
\hline Nausea & $7(11.48)$ & $12(19.67)$ & $\begin{array}{l}19 \\
(15.57)\end{array}$ & 0.212 \\
\hline Bedsore & $1(1.64)$ & - & $1(0.8)$ & $1.000+$ \\
\hline \multicolumn{5}{|l|}{ Chi-Square test } \\
\hline \multicolumn{5}{|l|}{ †Fisher's exact test } \\
\hline ‡Mann-Whitney U test & & & & \\
\hline
\end{tabular}

\section{Psychological Outcomes}

For Thai-HAD scores at baseline (day 1 of hospitalization), the majority of all enrolled patients had no anxiety, and depression, which the percentage were $59 \%$, and $53 \%$, respectively. Almost twenty percent of all enrolled patients had significant anxiety. Approximately one-fourth of all enrolled patients were facing with depression at day 1 of hospitalization. The median of anxiety-, and depression subscale at baseline 
were not different between two groups (Table 2). The median anxiety score for professional collaborative team group and medical oncologist group were similar, which score was 6 . Moreover, the median depressive score for professional collaborative team group and medical oncologist group at baseline were not statistically significant different, which depressive scores were 6 , and 9 , respectively ( $p$-value= 0.13) (Table 2).

Table 2

Thai-Hospital Anxiety and Depression Scale (Thai-HADS) at baseline (day 1 of hospitalization) between two groups

\begin{tabular}{|c|c|c|c|c|}
\hline & $\begin{array}{l}\text { Professional Collaborative Team ( } \mathrm{n} \\
=61)\end{array}$ & $\begin{array}{l}\text { Medical } \\
\text { Oncologists } \\
(n=61)\end{array}$ & Total & $\begin{array}{l}p- \\
\text { value }\end{array}$ \\
\hline & $\mathrm{n}(\%)$ & $\mathrm{n}(\%)$ & $\mathrm{n}(\%)$ & \\
\hline Anxiety & & & & 0.961 \\
\hline Absent & $36(59.02)$ & $36(59.02)$ & $72(59)$ & \\
\hline Yes, but normal & $13(21.31)$ & $14(22.95)$ & $\begin{array}{l}27 \\
(22.1)\end{array}$ & \\
\hline Yes, abnormal & $12(19.67)$ & $11(18.03)$ & $\begin{array}{l}23 \\
(18.8)\end{array}$ & \\
\hline $\begin{array}{l}\text { Median (Min - } \\
\text { Max) }\end{array}$ & $6(0-16)$ & $6(0-19)$ & & $0.734 \ddagger$ \\
\hline Depression & & & & 0.062 \\
\hline Absent & 39 (63.93) & $26(42.62)$ & $\begin{array}{l}65 \\
(53.2)\end{array}$ & \\
\hline Yes, but normal & $8(13.11)$ & $13(21.31)$ & $\begin{array}{l}21 \\
(17.2)\end{array}$ & \\
\hline Yes, abnormal & $14(22.95)$ & $22(36.07)$ & $\begin{array}{l}36 \\
(29.5)\end{array}$ & \\
\hline $\begin{array}{l}\text { Median (Min - } \\
\text { Max) }\end{array}$ & $6(0-18)$ & $9(0-21)$ & & $0.137 \ddagger$ \\
\hline \multicolumn{5}{|l|}{ Chi-Square test } \\
\hline \multicolumn{5}{|c|}{ ‡Mann-Whitney U test } \\
\hline \multicolumn{5}{|c|}{ Significant if $p<0.05$} \\
\hline
\end{tabular}

For Thai-HAD scores at 7 days of hospitalization, the median anxiety score for patients with professional collaborative team group was 5 , which score was lower than median anxiety score at day 1 of hospitalization significantly ( $p$-value $=0.003$ ). Additionally, the median depressive score for patients with professional collaborative team group was 5 , which score was lower than median depressive score at day 1 of hospitalization significantly ( $p$-value $<0.001$ ) (Fig. 2). The median anxiety score for patients with 
medical oncologist group at day 7 of hospitalization was 6 , which median score was not statistically significant different from day 1 ( $p$-value $=0.06)$. Meanwhile, the median depressive score for patient with medical oncologist group at day 7 was lower than score at day 1 of hospitalization significantly, which median score was 6 at day 7 when compared to score of 9 at day 1 of hospitalization $(p$-value $=0.006)$ (Table 3). However, the difference in the median of anxiety and depressive scores from day 1 to day 7 was not statistically significant different between two groups (Supplementary Table 1).

Table 3 Thai-Hospital Anxiety and Depression Scale (Thai-HADS) at day 1 and day 7 of hospitalization on each group

\begin{tabular}{|c|c|c|c|}
\hline & Day 1 & Day 7 & $p$-value \\
\hline \multicolumn{4}{|c|}{ Professional Collaborative Team } \\
\hline \multicolumn{4}{|l|}{ Anxiety } \\
\hline median (min - max) & $6(0-16)$ & $5(0-15)$ & 0.003 \\
\hline \multicolumn{4}{|l|}{ Depression } \\
\hline median (min - max) & $6(0-18)$ & $5(0-17)$ & $<0.001$ \\
\hline \multicolumn{4}{|l|}{ Medical Oncologists } \\
\hline \multicolumn{4}{|l|}{ Anxiety } \\
\hline median (min - max) & $6(0-19)$ & $6(0-19)$ & 0.068 \\
\hline \multicolumn{4}{|l|}{ Depression } \\
\hline median (min - max) & $9(0-21)$ & $6(0-19)$ & 0.006 \\
\hline \multicolumn{4}{|c|}{ Wilcoxon Signed Ranks test } \\
\hline Significant if $p<0.0$ & & & \\
\hline
\end{tabular}

\section{Quality Of Life Outcomes}

There was similar in Thai FACT-G score including physical-, social-, emotional-, and functional well-being scores at baseline (day 1 of hospitalization) between two groups ( $p$-value $=0.55$ ) (Supplementary Table 2).

For Thai FACT-G scores at 7 days of hospitalization, the median Thai FACT-G score for patients with professional collaborative team group was higher than scores at day 1 of hospitalization significantly including physical-, emotional-, functional well-being scores (Fig. 3). The median Thai FACT-G scores for patients with medical oncologist group at day 7 of hospitalization was not statistically significant different from day 1 ( $p$-value $=0.16)$, except physical-, and functional well-being subscale at day 7 were 
higher than day 1 of hospitalization significantly (Supplementary Table 3). The difference in the median of Thai FACT-G scores from day 1 to day 7 was not statistically significant different between two groups, except the difference in the median of social well-being subscale from day 1 to day 7 was significantly improved for patients with professional collaborative team group, when compared to patients with medical oncologist group $(p$-vale $=0.043)($ Supplementary Table 4, Supplementary Fig. 2).

\section{Quality Of Care Outcomes}

Re-admission rate at 7 days after hospital discharge was statistically significant reduced in patients with interprofessional collaborative team group when compared to patients with medical oncologist team group, which re-admission rate were 4.9\%, and 19.7\% ( $p$-value $=0.013$ ), respectively. Twelve of 61 patients with medical oncologist team were re-hospitalized at 7 days after hospital discharge, and only 3 of 61 patients with interprofessional collaborative team group. However, there was not statistically significant different in re-admission rate at 30 days after hospital discharge between two group, which re-admission rate were $32.8 \%$ in patients with interprofessional collaborative team group, and $40.9 \%$ in patients with medical oncologist team ( $p$-value $=0.34)$. Both groups were still alive at 30 days after hospital discharge, which the percentage was approximately $80 \%$. The median duration of hospitalization was longer in patients with interprofessional collaborative team group than patients with medical oncologist team significantly, which was 14 days (7-127 days), and 11 days $(7-45$ days) ( $p$-value $=0.01)$ (Supplementary Table 5).

\section{Discussion}

The aim of this study is to endorse that advanced solid cancer in-patients experience the best possible quality of life from palliative care in resource limited health care environment. Regarding to complex mechanisms and clinical presentations of suffering symptoms, including its physical, psychological, and spiritual aspects, multidisciplinary team approach is required for providing the effective palliative care. Moreover, the discordance between patients-reported and physician-documented symptoms was reported (5). One study shown that compared 58 questionaries completed by advanced cancer patients and their paired physician completed in medical records. The results showed that pain assessment was high concordance (96\%), but psychological and other aspects were discordance, which is associated with poor QoL, and distress (5-6).

In our limited resource condition, interprofessional team approach for this study was co-working in palliative care between specialist palliative care nurses, and medical oncologists. In fact, mood disorders are major contributors to morbidity in patients with advanced cancer (7). We considered using ThaiHospital Anxiety and Depression Scale (Thai-HADS), and Thai-Functional Assessment of Cancer TherapyGeneral (Thai FACT-G) scale as main measurement. The Thai-Hospital Anxiety and Depression Scale (Thai-HADS) is the most reliability and validity for mood assessment in palliative care setting. Moreover, the Thai-Functional Assessment of Cancer Therapy-General (Thai FACT-G) is the best tool for 
comprehensive quality of life assessments, including its physical, social, emotional, and functional wellbeings (2). Furthermore, palliative care assessment for hospitalized advanced cancer patients in the Netherland evaluated the quality of life by using EQ5D scores, which the questionnaires provide a simple, generic questionnaire for use in clinical and economic appraisal and population health surveys (8).

The team approach from our study explored the effect of having specialist palliative care nurses and medical oncologists in the palliative care team, which advanced cancer in-patients with team-based approach significantly improved anxiety-, and depressive subscale at day 7 of hospitalization, and clinical meaningfully improved quality of life in social well-being subscale. Our study results were similar to previous studies. For example, one randomized controlled trial showed improvement of depressive subscale significantly in advanced non-small cell lung cancer patients who were assessed palliative care by multidisciplinary team approach $(p$-value $=0.01)(2)$. In addition, the ENABLE trial shown that cancer patients who were received palliative care by specialist palliative care team had lower incidence of depression than patients who were received assessment by physicians $(p$-value $=0.02)$ regarding to focusing on coping skills and psychosocial concerns from specialist palliative care professionals (8). Another randomized controlled trial demonstrated that early palliative care by specialist palliative care team significantly improved depressive symptoms in advanced stage cancer patients $(p$-value $=0.01)$, and depressive symptom was associated with mortality (10). Additionally, Interestingly, one study demonstrated a positive correlation between quality of life of advanced cancer patients and their survival (11-14). Therefore, we hypothesize that improvement of mood and quality of life from palliative care in advanced cancer patients may prolong their survival. Further study should be explored.

There are a few studies focusing on readmissions for cancer patients. However, the re-admission rate is a crucial balancing measure to indicate a quality of palliative care and continuity of care, and healthcare resource allocation (15). Our study found that readmission rate was significantly reduced in patients who were received palliative care by team-based approach significantly. However, the median length of stay for patients who were assessed by our team-based approach were longer than patients who were assessed by medical oncologist team, which the median duration of hospital stay were 14 days (7-127 days), and 11 days (7-45days), respectively. Regarding to non-randomized study design, our provocative results would seem to conflict with existing theories that readmission rate is indication of quality issue related to shortened length of stay. In addition, patients who were assessed by our team-based approach with longer in length of hospital stay might indicate a relatively unstable condition.

Only approximately $10 \%$ on each group died during their admission for receiving palliative care, while the population-based cohort study from Taiwan shown that the majority of hospitalized cancer patients for palliative care (59\%) died during their first admission (16). This result may indicate that an early palliative care is associated with reduction of mortality rate during admission. Moreover, this study demonstrated that poor communication with patients and their family, inadequate outpatient follow-up and care coordination may attribute to increase readmission rate and mortality (16). 
This study had several limitations. First, the study design was non-randomization, therefore selection bias could be occurred due to an error in the procedure used to select target populations. Moreover, it could potentially occur self-selection bias because it is likely that their motivation for participation into interprofessional collaborative team group. Second, generalizability as this study was conducted in an institution. Third, there was short-term follow up of this study. Fourth, there was not ideally multidisciplinary team-based approach regarding limited resource in human reason. Fifth, we did not distinguish subclassification of advanced stage to terminal stage of disease. To address the limitations, the randomization method eliminates the selection bias, and balances the groups with respect to many confounding variables. Furthermore, multicenter study and longer follow up time are required.

\section{Conclusion}

Our study concluded that co-working and the communication between specialist palliative care nurses and medical oncologists as team-based approach is considered to be a key factor for effective interprofessional collaboration in a resource limited setting. Advanced cancer patients who were assessed palliative care with team-based approach improved their either quality of life or psychological aspects, and significantly reduce readmission rate at 7 days after hospital discharge. The results may help inform policy discussions to improve the quality of palliative care and to reduce the readmission rate among advanced cancer inpatients.

\section{Declarations}

Funding:This study received funding from Department of Medicine, Phramongkutklao Hospital, Bangkok, Thailand.

Conflicts of interest: The authors certify that they have NO affiliations with or involvement in any organization or entity with any financial (such as honoraria; educational grants; participation in speakers' bureaus; membership, employment, consultancies, stock ownership, or other equity interest; and expert testimony or patent-licensing arrangement), or non-financial interest (such as personal or professional relationships, affiliations, knowledge or beliefs) in the subject matter or materials discussed in this manuscript.

Availability of data and material: The authors confirm that the data supporting the findings of this study are available within the article and its supplementary materials.

Code availability:Code for data cleaning and analysis is provided as aprt of the replication package. It is available at https://www.dropbox.com/s/s3vp276a9bpfbwg/Code\%20and\%20data_Palliative$30042021 \% 20 \% 281 \% 29 . x \mid s x$ ?dl $=0$.

Authors' contributions:All authors contributed to the study conception and design. Material preparation, data collection and analysis were performed by PitchayapaPornrattanakavee, TassayaSrichan , and Naiyarat Prasongsook. The first draft of the manuscript was written by 
PitchayapaPornrattanakavee,Naiyarat Prasongsook, and all authors commented on previous versions of the manuscript. All authors read and approved the final manuscript.

Ethics approval:This study was conducted according to ICH-GCP guidelines and the Declaration of Helsinki. The study protocol was approved by Institutional Review Boards, Royal Thai Army Medical Department (protocol number: R008h/63). Informed consent was obtained from all individual participants included in the study.

Consent to participate: All patients provided written informed consent for trial participation. This study is registered under Thai Clinical Trials Registry (TCTR) 20200312001.

Consent for publication: Participants signed informed consent regarding publishing their data.

Acknowledgements:The authors are grateful to the Office of research Development, Phramongkutkao Hospital, and PMK Cancer Center for supporting this study.

\section{References}

1. World Health Organization. 2020 [Internet]. [cited 2020Feb12]. Available from: https://www.who.int/health-topics/palliative-care

2. Ferrell BR, Temel JS, Temin S, et al. Integration of Palliative Care IntoStandard Oncology Care: American Society of Clinical Oncology ClinicalPractice Guideline Update. J Clin Oncol 2017;35:96112.

3. Isenberg SR, Lu C, McQuade J, et al. Economic Evaluation of a Hospital-Based Palliative Care Program. J Oncol Pract 2017;13:e408-e420.

4. Isenberg SR, Lu C, McQuade J, et al. Impact of a New Palliative CareProgram on Health System Finances: An Analysis of the Palliative CareProgram Inpatient Unit and Consultations at Johns Hopkins Medicallnstitutions. J Oncol Pract 2017;13:e421-e430.

5. Strömgren AS, Groenvold M, Pedersen L, et al. Does the medical record cover thesymptoms experienced by cancer patients receiving palliative care? A comparison of therecord and patient selfrating. J Pain Symptom Manage 2001; 21:189.

6. Chandwani KD, Zhao F, Morrow GR, et al. Lack of Patient-Clinician Concordance in CancerPatients: Its Relation With Patient Variables. J Pain Symptom Manage 2017; 53:988.

7. Maione P, Perrone F, Gallo C, et al. Pretreatment quality of life and functional status assessment significantly predict survival of elderly patients with advanced non-small-cell lung cancer receiving chemotherapy: a prognostic analysis of the Multicenter Italian Lung Cancer in the Elderly Study. J Clin Oncol 2005;23:6856-72.

8. Bakitas M, Lyons KD, Hegel MT, et al. Effects of a palliative care intervention on clinical outcomes in patients with advanced cancer: The Project ENABLE II randomized controlled trial. JAMA. 2009;302: 741-749. 
9. Clinical practice guidelines for quality palliative care, 4th ed. Pittsburgh: National Consensus Project, 2009. (Assessed July,14, 2019, at www.nationalcoalitionhpc.org/ncp.)

10. Nickolich MS, El-Jawahri A, Temel J, et al. Discussing the evidence for upstream palliative care in improving outcomes in advanced cancer. American Society of Clinical Oncology Educational Book, 2016,(36):534-538.

11. Grace M, Ravindran K, Shirlyn $\mathrm{H}$, et al. Pilot study of a palliative care and medical oncology corounding model for advanced cancer inpatient in a tertiary hospital in Singapore. J Palliative med 2018;21:95-98.

12. Yoong J, Park ER, Greer JA, et al. Early palliative care in advanced lung cancer: a qualitative study. JAMA Intern Med. 2013;173:283-290

13. Prescott A, Hull J, Dionne-Odom N, et al. The role of a palliative care intervention in moderating the relationship between depression and survival among individuals with advanced cancer. Health Psychol 2017,36(12): 1140-114.

14. Park S.A, Chung S, Lee Y, et al. Factors influencing the quality of life of patients with advanced cancer. Applied Nursing Research 2016; 33:108-112.

15. Stoppelenburga AB, Vergouwea $Y$, Mathijs MB, et al. The Impact of Palliative Care Team Consultation on Quality of Life of Patients with Advanced Cancer in Dutch Hospitals: An Observational Study.Oncol Res Treat 2020;43:405-413.

16. Hsiao-Ting Chang, Chun-Ku Chen, Ming-Hwai Lin, et al. Readmissions in cancer patients after receiving inpatient palliative care in Taiwan: A 9-year nationwide population-based cohort study. Medicine 2016; 8 (95): 2782-2788.

\section{Figures}




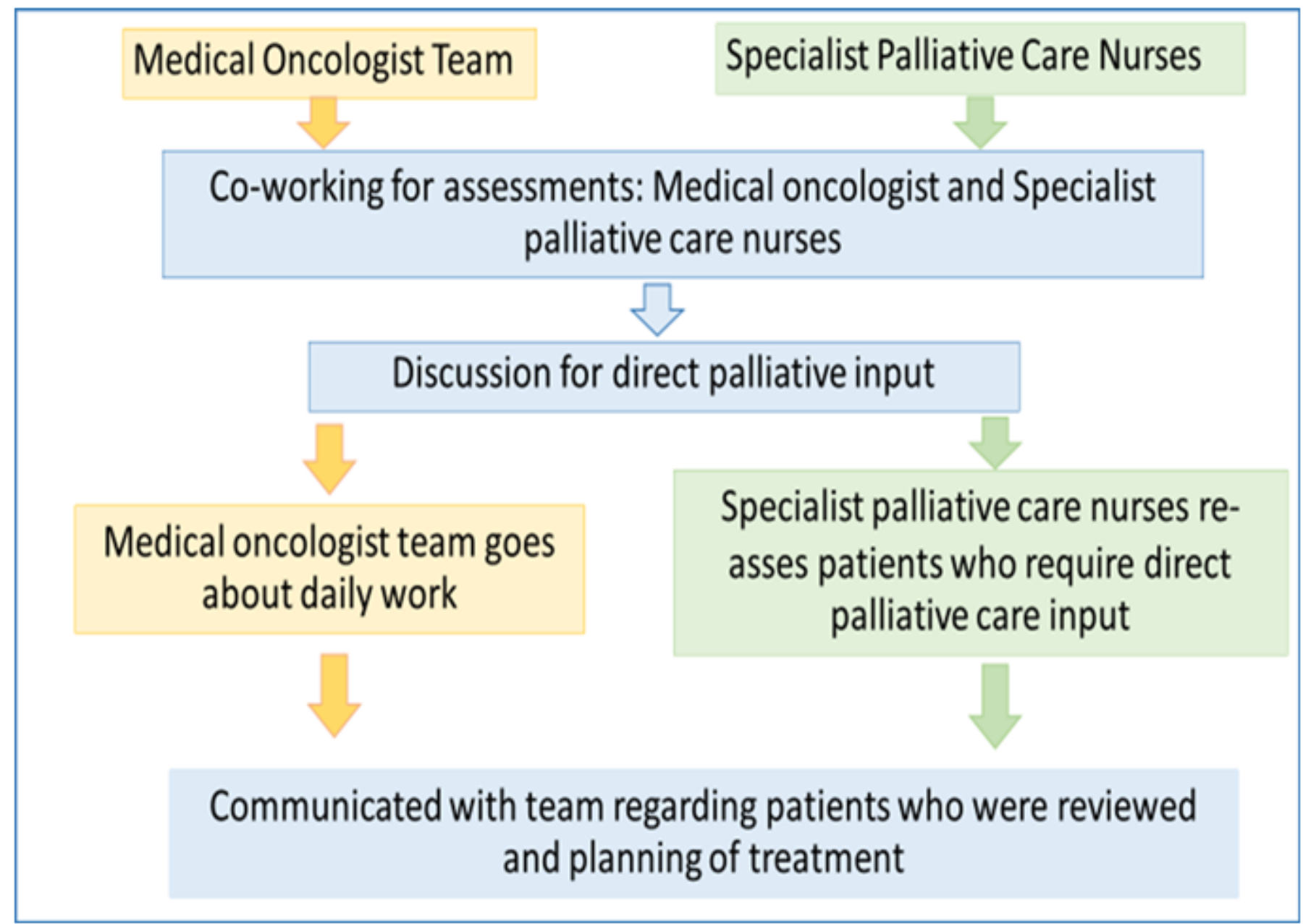

Figure 1

Schema of co-working for palliative care between specialist palliative care nurses and medical oncologists as interprofessional collaborative team 


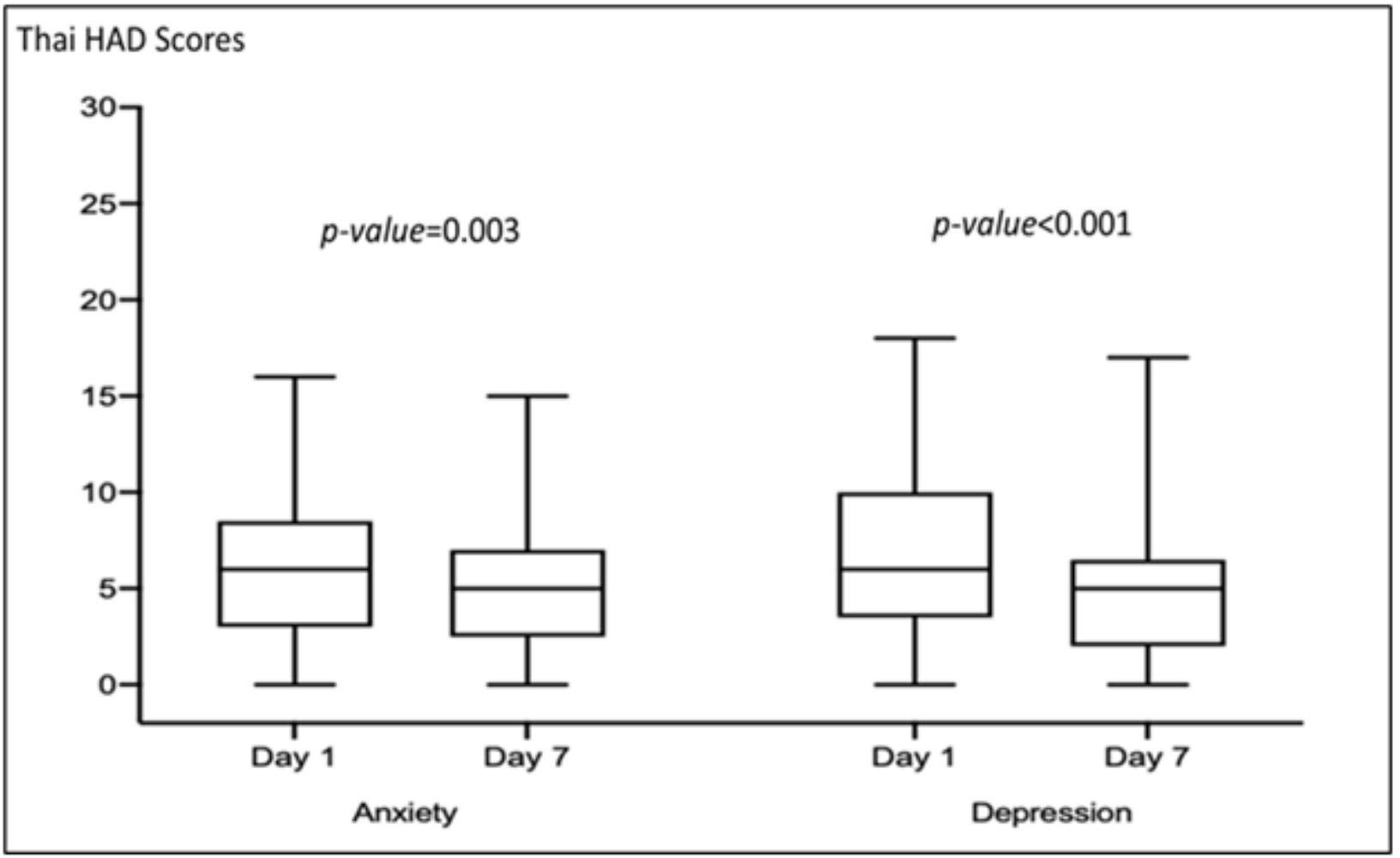

Figure 2

Thai-Hospital Anxiety and Depression Scale (Thai-HADS) at day 1 and day 7 of hospitalization in Professional Collaborative Team

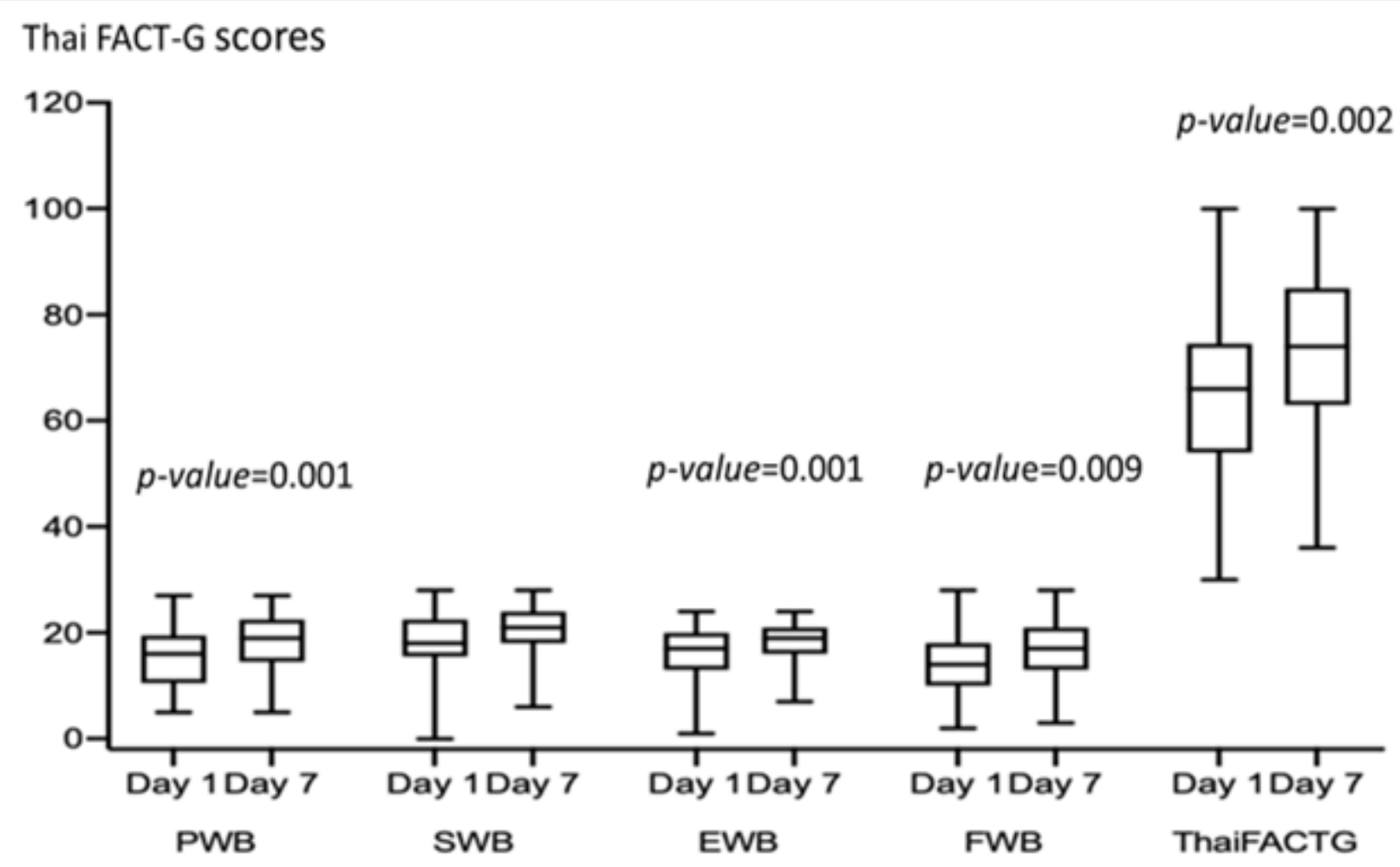


Figure 3

Thai-Functional Assessment of Cancer Therapy-General (Thai FACT-G) scale at day 1 and day 7 of hospitalization in interprofessional collaborative Team

\section{Supplementary Files}

This is a list of supplementary files associated with this preprint. Click to download.

- SupplementaryFiguresandtables.docx 